\title{
High mobility group box 1 regulates tumor metastasis in cutaneous squamous cell carcinoma via the PI3K/AKT and MAPK signaling pathways
}

\author{
YING SUN ${ }^{1}$, YING TU ${ }^{2}$, $\mathrm{LI} \mathrm{HE}^{2}, \mathrm{CHAO} \mathrm{JI}^{3}$ and $\mathrm{BO} \mathrm{CHENG}^{3}$ \\ ${ }^{1}$ Department of Dermatology, The First Affiliated Hospital of Wenzhou Medical University, Wenzhou, Zhejiang 325035; \\ ${ }^{2}$ Department of Dermatology, The First Affiliated Hospital of Kunming Medical University, \\ Yunnan Provincial Institute of Dermatology, Kunming, Yunnan 650032; \\ ${ }^{3}$ Department of Dermatology, The First Affiliated Hospital of Fujian Medical University, \\ Fuzhou, Fujian 350005, P.R. China
}

Received October 23, 2014; Accepted August 17, 2015

DOI: $10.3892 / 01.2015 .3843$

\begin{abstract}
The present study examined the mechanisms of high mobility group box 1 (HMGB1)-induced cell migration in human cutaneous squamous cell carcinoma (CSCC) SCC13 cells. Western blotting, a chemotaxis assay and ELISA were performed to analyze HMGB1 level in SCC13 cells and its ability to regulate tumor metastasis. The results demonstrated a significantly higher level of HMGB1 in the SCC13 cell supernatant compared with the human epidermoid carcinoma A431 cell supernatant. Administration of HMGB1 to the SCC13 cells caused cell migration, which occurred in a time- and dose-dependent manner. Moreover, HMGB1 significantly activated the phosphosphoinositide 3-kinase (PI3K)/AKT and mitogen-activated protein kinase (MAPK) signaling pathways by an increased level of phosphorylation in p85PI3K, AKT, p38 and p42/44 MAPK. Taken together, these data suggest that HMGB1 regulates tumor metastasis in CSCC via the PI3K/AKT and MAPK signaling pathways.
\end{abstract}

Correspondence to: Dr Ying Sun, Department of Dermatology, The First Affiliated Hospital of Wenzhou Medical University, 3 Fuxue Road, Wenzhou, Zhejiang 325035, P.R. China E-mail: avision718@sina.com

Dr Bo Cheng, Department of Dermatology, The First Affiliated Hospital of Fujian Medical University, 20 Cha Zhong Road, Fuzhou, Fujian 350005, P.R. China

E-mail: chengbo_fjmu@sina.com

Abbreviations: CSCC, cutaneous squamous cell carcinoma; HMGB1, high-mobility group box 1; MAPK, mitogen-activated protein kinase; PI3K, phosphoinositide 3-kinase; GR, glycyrrhizin; ELISA, enzyme-linked immunosorbent assay

Key words: cutaneous squamous cell carcinoma, HMGB1, migration, PI3K/AKT pathway, MAPK pathway

\section{Introduction}

Skin cancer is the most frequently occurring among all types of human cancers, and cutaneous squamous cell carcinoma (CSCC) is the second most common of all the skin tumors (1). CSCC is a major health concern in Caucasian in the United States, with a similar mortality rate to melanoma (2). In total, $>700,000$ new cases of CSCC are diagnosed every year, and this assumes that $20 \%$ of non-melanoma skin cancers (NMSCs) are CSCCs (3). CSCC is a type of tumor that is more aggressive than other skin carcinomas, as $12 \%$ of cases metastasize $(4,5)$. Generally, the prognosis of CSCC is satisfactory, however, $4 \%$ of cases will still develop metastases and $1.5 \%$ of patients will succumb to this disease $(6,7)$. The rising incidence and morbidity rates of $\mathrm{CSCC}$ have generated great interest for researchers with regard to the progression and mechanisms of metastatic CSCC migration to the distant organs.

High-mobility group box 1 protein (HMGB1), a chromatin-binding nuclear protein, is highly expressed in numerous types of cancer cells and is involved in cell progression (8). Besides its intracellular function, HMGB1 can also act as an extracellular molecule binding to the receptor for advanced glycation end products and toll-like receptors, therefore resulting in cell differentiation, cell migration, tumor progression and inflammation $(9,10)$. The function of extracellular HMGB1 is important in the metastasis of several types of tumor cells (11).

However, the role of HMGB1 in the migration of CSCC remains largely uninvestigated (12). We hypothesize that HMGB1 is a key regulator that induces metastatic CSCC to migrate. To investigate this, the present study compared CSCC cells with human epidermoid carcinoma cells in order to assess the level of HMGB1. Next, the study determined whether HNGB1 was able to induce migration in CSCC and investigated the signaling pathway involved in this process, so that it could be determined whether HMGB1 is a potential therapeutic target for preventing the metastasis of CSCC. 


\section{Materials and methods}

Cell lines and culture. Human CSCC SCC13 and epidermoid carcinoma A431 cells were purchased from the American Type Culture Collection (Manassas, VA, USA). The two cell lines were cultured in Iscove's modified Dulbecco's medium supplemented with $10 \%$ heat-inactivated fetal bovine serum, $2 \mathrm{mM}$ glutamine and an antibiotic-antifungal mixture in a humidified incubator with $5 \% \mathrm{CO}_{2}$ and $95 \%$ air. Each cell line was confirmed according to the American Type Culture Collection instructions.

Antibodies and reagents. Recombinant HMGB1 protein was obtained from Eli Lilly Company (Indianapolis, IN, USA) and glycyrrhizin (GR) was obtained from Sigma-Aldrich, (St. Louis, MO, USA). The antibodies for the western blotting were rabbit monoclonal anti-phospho-akt (Thr308; catalog no. C31E5E; 1:1,000 dilution), rabbit monoclonal anti-phospho-PI3kinase p85 [(Tyr458)/p55 (Tyr199); 1:1,000 dilution)], rabbit monoclonal anti-phospho-p38 MAPK (Thr180/Tyr182; catalog no. 3D7; 1:1,000 dilution), mouse monoclonal anti-phospho-p44/42 MAPK (Erk1/2) (Thr202/ Tyr204; catalog no. E10; 1:1,000 dilution), rabbit monoclonal anti-AKT (1:1,000 dilution), rabbit monoclonal anti-PI3kinase p85 (1:1000 dilution), rabbit monoclonal anti-p38 MAPK (1:1,000 dilution) and rabbit monoclonal anti-p42/44 MAPK (1:1,000 dilution). These antibodies were obtained from Cell Signaling Technology (Danvers, MA, USA).

Western blotting. Briefly, after treatment with/without HMGB1 or GR, the whole cell lysates were prepared with ice- cold cell lysis buffer and cleared by centrifugation at $10,000 \mathrm{x} \mathrm{g}$. The total protein concentration was measured with the bicinchoninic acid assay kit (Bio-Rad Laboratories, Inc., Hercules, CA, USA). Equal amount of proteins were loaded onto 8-12\% SDS-PAGE gel sand transferred to polyvinylidene fluoride membranes by electroblotting. Subsequent to blocking in phosphate-buffered saline (PBS) plus Tween-20 containing 5\% dried milk at room temperature for $1 \mathrm{~h}$, the membranes were incubated with primary antibodies at $4^{\circ} \mathrm{C}$ overnight. The blots were incubated with appropriate secondary antibodies at room temperature for $1 \mathrm{~h}$ the next day. The signals were detected by enhanced chemiluminescence reagent (ThermoFisher Scientific, Inc., Rockford, IL, USA).

Chemotaxis assay. The 8- $\mu \mathrm{m}$ polycarbonate membranes were incubated with $5 \mu \mathrm{g}$ fibronectin in $400 \mu \mathrm{l}$ PBS overnight at $4^{\circ} \mathrm{C}$ and blocked with $1 \%$ bovine serum albumin for $2 \mathrm{~h}$ prior to use. Briefly, the cells were detached with trypsin, washed twice with PBS and resuspended in serum-free Dulbecco's modified Eagle's medium to a density of $5 \times 10^{5}$ cells $/ \mathrm{ml}$. Next, $200 \mu \mathrm{l}$ of the prepared cells were seeded into the upper chambers of Transwell inserts (Costar Transwell; Corning Inc., Corning, NY, USA). The lower chambers were filled with different concentrations of HMGB1 (10,30 and $100 \mathrm{ng} / \mathrm{ml})$, as indicated, or with DMEM (control). After $12 \mathrm{~h}$ of incubation at $37^{\circ} \mathrm{C}$, the inserts were removed from the Transwells and thoroughly washed three times with PBS. Cells remaining in the upper chambers were wiped off with swabs and cells that had migrated were stained by Hema 3 according to the
A

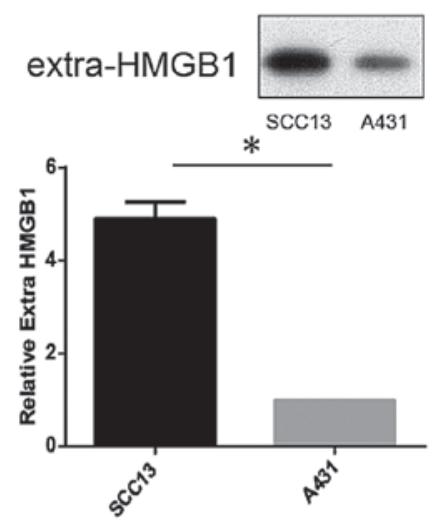

B

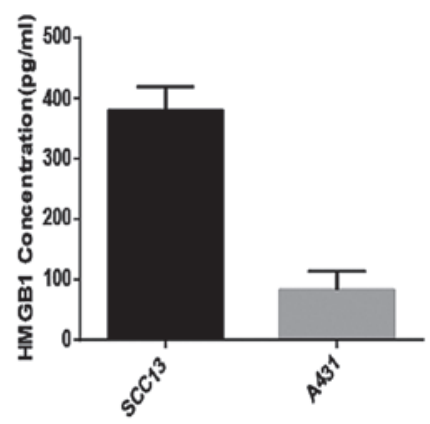

Figure 1. CSCC SCC13 cells secrete more HMGB1 than human epidermoid carcinoma A431 cells. (A) Human CSCC SCC13 and epidermoid carcinoma A431 cells were cultured with regular medium for $24 \mathrm{~h}$. Following this, concentrated media was prepared, resolved by SDS-PAGE and subjected to western blot analysis of HMGB1. Results shown are representative of three independent experiments and were qualified by densitometry. ${ }^{\mathrm{P}} \mathrm{P}<0.01$. (B) Enzyme-linked immunosorbent assay data of the supernatant showing the HMGB1 concentration in the two different cell lines. Data from three independent experiments were pooled together. CSCC, cutaneous squamous cell carcinoma; HMGB1, high-mobility group box 1 .

manufacturer's instructions (Thermo Fisher Scientific, Inc., Waltham, MA, USA). The adherent cells were then counted under a light microscope (Olympus BX63 Upright Microscope; Olympus Corporation, Tokyo, Japan).

Enzyme-linked immunosorbent assay (ELISA). Levels of HMGB1 in the supernatant of the samples were measured using the human HMGB1 ELISA assay kit (Immuno-Biological Laboratories International, Toronto, ON, Canada) according to the manufacturer's instructions.

Statistical analysis. Data in this study are presented as the mean \pm standard error of the mean of at least three different independent experiments. The statistical analysis was performed using GraphPad Prism software, version 6.0 (GraphPad Software, Inc., La Jolla, CA, USA). Results were analyzed by Student's t-test for comparisons between the two groups. $\mathrm{P}<0.05$ was considered to indicate a statistically significant difference.

\section{Results}

Human CSCC SCC13 cells secrete higher HMGB1 levels compared with other NMSC cells. Since HMGB1 is well known as an important regulator in the development, progression and 
A

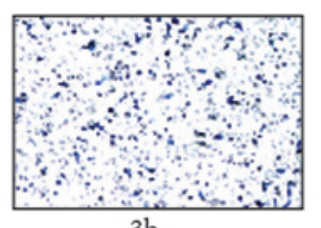

$3 \mathrm{~h}$

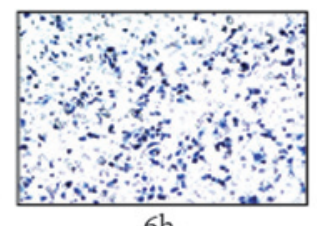

$6 \mathrm{~h}$

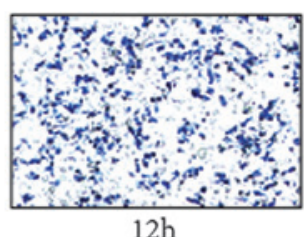

$12 \mathrm{~h}$

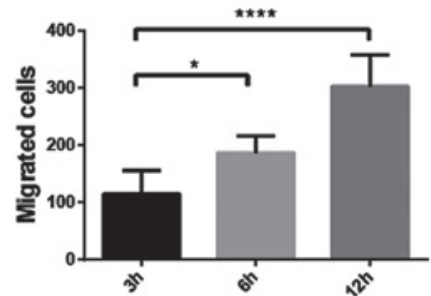

Time
B

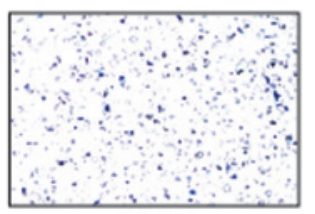

SFM

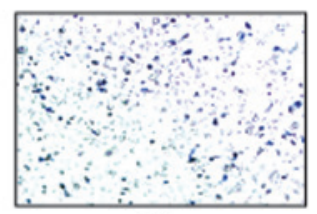

10

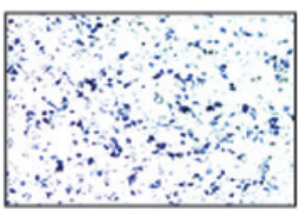

30

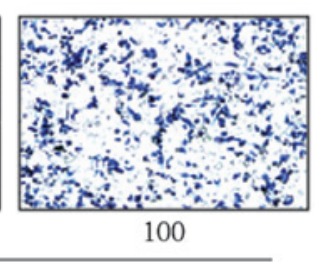

$\mathrm{HMGB} 1(\mathrm{ng} / \mathrm{ml})$

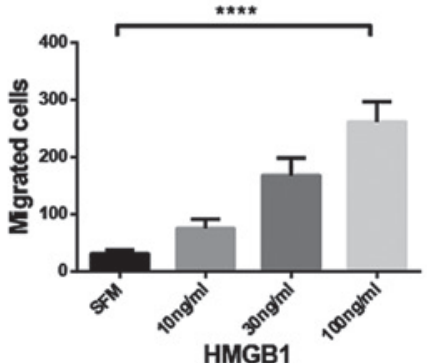

HMGB1

Figure 2. HMGB1 induces the cell migration of human CSCC cells. (A) CSCC SCC13 cells were treated for the indicated times ( 3,6 and 12 h). The number of migrated cells were counted and the results expressed as the mean number of migrated cells \pm SEM/microscopic field ( $=3$ ). A significantly higher level of cell migration was found at the 12 -h time-point $\left({ }^{*} \mathrm{P}<0.05,{ }^{* * * * *} \mathrm{P}<0.0001\right)$. (B) The SCC13 cells were treated with the indicated concentration of HMGB1 for $12 \mathrm{~h}$. The number of migrated cells was counted and the results expressed as the mean number of migrated cells $\pm \mathrm{SEM} / \mathrm{microscopic}$ field ( $\mathrm{n}=3$ ). A significantly higher level of cell migration was found at a HMGB1 concentration of $100 \mathrm{ng} / \mathrm{ml}\left({ }^{* * * *} \mathrm{P}<0.0001\right)$. CSCC, cutaneous squamous cell carcinoma; HMGB1, high-mobility group box 1 .

A
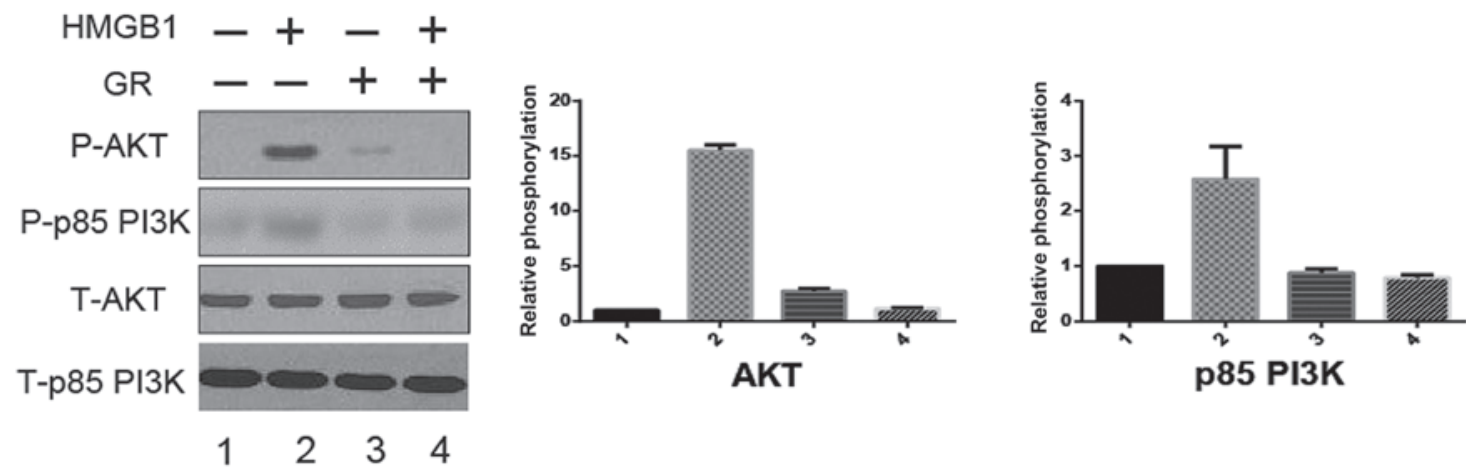

$\mathbf{B}$
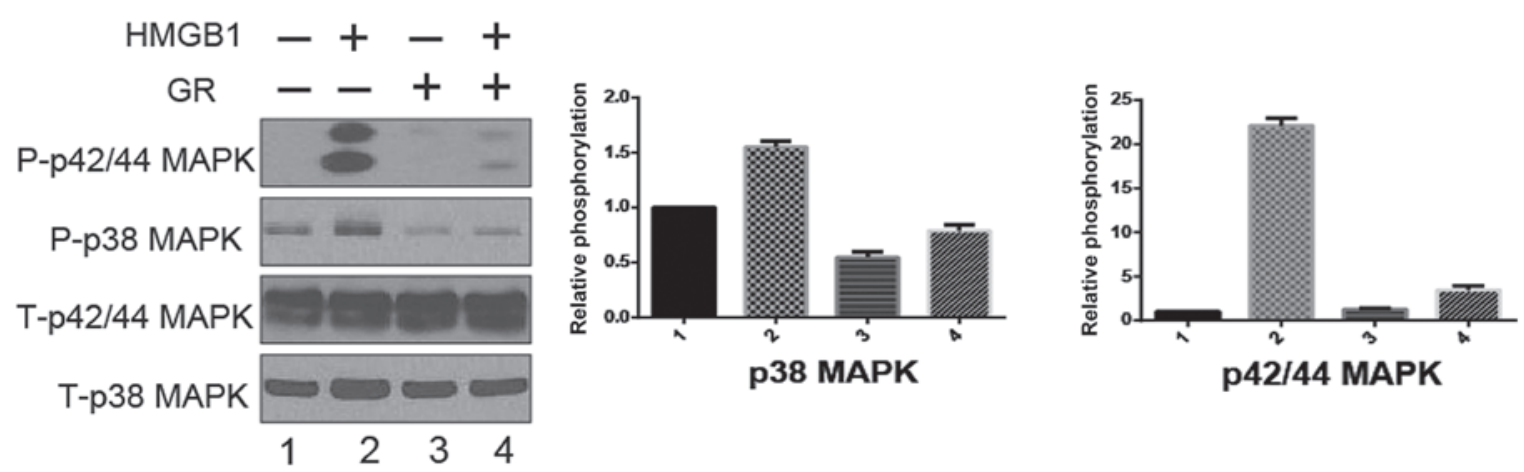

Figure 3. PI3K/AKT and MAPK signaling pathways are involved in HMGB1-induced migration. CSCC SCC13 cells were treated with/without HMGB1 $(100 \mathrm{ng} / \mathrm{ml})$ or GR $(100 \mu \mathrm{M})$ for $24 \mathrm{~h}$. At the end of treatment, the activation [phosphorylation (P)] of AKT, p85 PI3K, p38 and p42/44 MAPK was analyzed by western blotting. The whole cell lysates were prepared, resolved by SDS-PAGE and subjected to western blot analysis. The total (T)-AKT, -p85 PI3K, -p38 and -p42/44 MAPK were used as a loading control. Results shown are representative of three independent experiments and were qualified by densitometry. (A) AKT and p85 PI3K; (B) p38 MAPK and p42/44 MAPK. CSCC, cutaneous squamous cell carcinoma; HMGB1, high-mobility group box 1; PI3K, phosphoinositide 3-kinase; MAPK, mitogen-activated protein kinase; GR, glycyrrhizin.

metastasis of certain types of cancers, the present study aimed to identify whether human CSCC SCC13 cells can secrete this protein, and the difference between SCC13 and other NMSC cells, namely A431 cells $(9,10)$. First, these two human cell lines were cultured in regular medium for $24 \mathrm{~h}$, then the supernatant was collected and a western blotting assay was performed 
(Fig. 1A). The level of HMGB1 in the supernatant of the SCC13 cells was significantly higher than the level in the A431 cell supernatant $(\mathrm{P}<0.01$; Fig. 1A). ELISA was also used to confirm these results (Fig. 1B). The levels of HMGB1 in the supernatant of these two cell lines were measured by the human HMGB1 ELISA assay kit. The human CSCC SCC13 cells secreted higher HMGB1 levels compared with the A431 cell line.

$H M G B 1$ regulates the metastatic potential of SCC13 cells in a time- and dose-dependent manner. First, the SCC13 cells were treated with HMGB1 $(100 \mathrm{ng} / \mathrm{ml})$ for different lengths of time (3, 6 and $12 \mathrm{~h}$ ), and then the number of migrated cells was counted under a microscope. A significantly higher level of cell migration was evident following incubation for $3 \mathrm{~h}$, however, the highest level was at the 12-h time-point ( $\mathrm{P}<0.0001$; Fig. 2A). Therefore, the 12-h time-point was chosen for further study. The SCC13 cells were next treated with different concentrations of HMGB1 for $12 \mathrm{~h}$. A significantly higher level of cell migration was evident at $100 \mathrm{ng} / \mathrm{ml}$ HMGB1 ( $<<0.0001$; Fig. 2B). These data indicated that an increase in HMGB1 level induces SCC13 cell migration in a time- and dose-dependent manner.

PI3K/AKT and MAPK signaling pathways are involved in HMGB1-induced migration. Since the PI3K/AKT and MAPK signaling pathways are well known to play an essential role in regulating cell proliferation, differentiation, migration and the other cell progression, the present study analyzed the PI3K/AKT and MAPK signals induced by HMGB1. The SCC13 cells were treated with HMGB1 (100 ng/ml) in the presence or absence of GR $(100 \mu \mathrm{M})$, which is an HMGB1 inhibitor, for $24 \mathrm{~h}$. It was found that HMGB1 stimulated the activation of the p38, p42/44 MAPK, AKT and PI3K signals in the SCC13 cells by an increased level of phosphorylated p38, P42/44 MAPK, AKT and PI3K. GR was able to almost completely inhibit this activation (Fig. 3), which indicates a key role of the AKT/AMPK pathway in regulating HMGB1-induced metastasis.

\section{Discussion}

CSCC is the second most commonly occurring type of NMSC, with an incidence rate that is increasing rapidly (13). The metastases of cancer cells are viewed as a major cause of mortality in the majority of cancer types. Since the metastasis of the primary cancer causes damage in other organs, treatment is no longer efficient (14). Metastatic CSCC causes the majority of fatalities associated with NMSC, but the molecular mechanisms for CSCC progression and migration remain poorly identified. The present study aimed to determine the element that plays an essential role in this progression and to unravel the associated signaling pathways.

HMGB1 is well known for its chemotactic activity in certain tumor cells (8-11), however, little is known with regard to its function in the metastasis of CSCC. Therefore, the present study sought to determine whether HMGB1 plays a role in this process. First, the level of HMGB1 was examined in CSCC cells and compared with normal skin carcinoma cells. It was found that the level of HMGB1 in the supernatant of the CSCC cells was significantly higher than that in the A431 cells, which meant that HMGB1 may be involved in this process. Next, the effects of HMGB1 on the migration of the CSCC cells were examined. The CSCC cells were treated with HMGB1 at different concentrations and for varying times. The results suggested that HMGB1 can induce the migration of CSCC cells in a time- and dose-dependent manner. In addition, it was found that the PI3K/AKT and MAPK signaling pathways are involved in the regulation of the HMGB1-induced migration of the CSCC cells (Fig. 3). Additional studies are required to clarify the downstream genes with critical roles.

Overall, the present study showed that HMGB1 is secreted more in CSCC cells than in human epidermoid carcinoma cells, and that HMGB1 regulates the migration of CSCC cells by activating the PI3K/AKT and MAPK signaling pathways. These results provide convincing evidence that the inhibition of HMGB1 and this pathway could have a strong effect on preventing the metastasis of CSCC. More detailed studies are required to determine whether the inhibitor of HMGB1 can be a pharmacologically safe and efficient agent for the treatment of CSCC.

\section{Acknowledgements}

This study was supported by grants from the National Natural Science Foundation of China (nos. 30860257, 81101188 and 810701297).

\section{References}

1. Uribe P and Gonzalez S: Epidermal growth factor receptor (EGFR) and squamous cell carcinoma of the skin: Molecular bases for EGFR-targeted therapy. Pathol Res Pract 207: 337-342, 2011.

2. Karia PS, Han J and Schmults CD: Cutaneous squamous cell carcinoma: Estimated incidence of disease, nodal metastasis and deaths from disease in the United States, 2012. J Am Acad Dermatol 68: 957-966, 2013.

3. Rogers HW, Weinstock MA, Harris AR, et al: Incidence estimate of nonmelanoma skin cancer in the United States, 2006. Arch Dermatol 146: 283-287, 2010.

4. Cherpelis BS, Marcusen C and Lang PG: Prognostic factors for metastasis in squamous cell carcinoma of the skin. Dermatol Surg 28: 268-273, 2002.

5. Czarnecki D, Staples M, Mar A, Giles G and Meehan C: Metastases from squamous cell carcinoma of the skin in southern Australia. Dermatology 189: 52-54, 1994.

6. Mourouzis C, Boynton A, Grant J, et al: Cutaneous head and neck SCCs and risk of nodal metastasis-UK experience. J Craniomaxillofac Surg 37: 443-447, 2009.

7. Brantsch KD, Meisner C, Schonfisch B, et al: Analysis of risk factors determining prognosis of cutaneous squamous-cell carcinoma: A prospective study. Lancet Oncol 9: 713-720, 2008.

8. Tang D, Kang R, Livesey KM, Zeh HJ III and Lotze MT: High mobility group box 1 (HMGB1) activates an autophagic response to oxidative stress. Antioxid Redox Signal 15: 2185-2195, 2011.

9. Tang D, Kang R, Cheh CW, et al: HMGB1 release and redox regulates autophagy and apoptosis in cancer cells. Oncogene 29: 5299-5310, 2010.

10. Lotze MT and Tracey KJ: High-mobility group box 1 protein (HMGB1): Nuclear weapon in the immune arsenal. Nat Rev Immunol 5: 331-342, 2005.

11. Penzo M, Molteni R, Suda T, et al: Inhibitor of NF-kappa B kinases alpha and beta are both essential for high mobility group box 1-mediated chemotaxis (corrected). J Immunol 184: 4497-4509, 2010.

12. Lambert SR, Harwood CA, Purdie KJ, et al: Metastatic cutaneous squamous cell carcinoma shows frequent deletion in the protein tyrosine phosphatase receptor Type D gene. Int J Cancer 131: E216-E226, 2012.

13. Madan V, Hoban P, Strange RC, Fryer AA and Lear JT: Genetics and risk factors for basal cell carcinoma. Br J Dermatol 154 (Suppl 1): 5-7, 2006.

14. Ekblad L and Johnsson A: Cetuximab sensitivity associated with oxaliplatin resistance in colorectal cancer. Anticancer Res 32: 783-786, 2012. 